\title{
CHROMOSOME VARIATION FOR LOCI CONTROLLING EAR EMERGENCE TIME ON CHROMOSOME 5A OF WHEAT
}

\author{
J. W. SNAPE, C. N. LAW and A. J. WORLAND \\ Plant Breeding Institute, Trumpingtan, Cambridge CB2 $2 L Q$
}

Received 13.iii.76

\begin{abstract}
Summary
Substitutions of chromosome 5A from different varieties into the variety Chinese Spring had differential effects on the ear emergence time of this variety. A model of three genetic factors was capable of explaining the genetical relationships between these chromosomes but it was not possible to distinguish whether these were three separate loci or three separate components of the Vrn 1 locus, located on this chromosome. The results suggest, however, that close adaptation of ear emergence times and vernalisation requirement is possible by genetical manipulation of this variation.
\end{abstract}

\section{INTRODUGTION}

InVESTIGATIONS of the genetical control of ear emergence time in bread wheat, Triticum aestivum $(2 n=6 x=42)$ using monosomic analysis on the one hand and chromosome substitution lines on the other, have shown that major effects are associated with chromosomes 5A and 5D (Unrau, 1950; Kuspira and Unrau, 1957; Halloran and Boydell, 1967; Law, 1968, 1972; Maystrenko, 1974). These same investigations have shown that these effects are synonymous with the response to vernalisation treatments; these chromosomes being important determinants of the spring-winter polymorphism of wheat.

Conventional genetic analysis by Akerman and Mackey (1949) and Pugsley $(1971,1972)$ have shown that spring habit is controlled by at least four independent, dominant loci with large effects, any one of which is able to reduce the requirement for vernalisation. These loci have been designated $V r n$ 1, 2, 3 and 4 and the aneuploid analyses have equated $V r n 1$ and $V r n 3$. with the effects of chromosomes $5 \mathrm{~A}$ and $5 \mathrm{D}$, respectively.

More detailed analysis of the genetical control of ear emergence time using substitutions of chromosomes $5 \mathrm{~A}$ and $5 \mathrm{D}$ from the variety Hope into the variety Chinese Spring by Law, Worland and Giorgi (1976) showed that a single factor located distally on the long arm of both chromosomes was responsible for the variation between these substitution lines and the recipient. They equated these factors with the Vrn 1 and Vrn 3 loci. However they also found that substitutions of chromosome $5 \mathrm{~A}$ from other varieties into Chinese Spring had differential effects on the ear emergence time of this variety. Either, therefore, a multiple allelic series exists at the $V r n 1$ locus, or more than one factor controlling ear emergence time is carried on this chromosome, these being homozygous with respect to the Hope/Chinese Spring difference.

The present paper presents a more detailed analysis of the variation between these different $5 \mathrm{~A}$ chromosomes and examines the genetical relationship between them. 


\section{Materials AND Methods}

Substitution lines of chromosome 5A from the varieties CappelleDesprez, Hope, Timstein and Triticum spelta into Chinese Spring were chosen for investigation. These substitution lines represent a range of ear emergence times, earlier and later than euploid Chinese Spring. The CappelleDesprez and T. spelta substitution lines were developed at the Plant Breeding Institute and the Hope and Timstein substitution lines by $\mathrm{Dr}$ E. R. Sears at the University of Missouri, U.S.A.

The four substitution lines plus Chinese Spring were crossed reciprocally in all combinations and selfed to produce a $5 \times 5$ diallel.

The $\mathrm{F}_{1}$ progeny of these crosses was sown in a randomised block design in a constant environment room. One plot of four plants of each parental line and one plot of four plants of each reciprocal cross were grown in each of two blocks. The plants were grown under a regime of continuous light at $20^{\circ} \mathrm{C}$ and ear emergence times recorded.

\section{Results}

Variation between blocks and reciprocal crosses and block interactions was found to be non-significant, so that in the analyses presented the data have been averaged over reciprocals and blocks and the sums of squares of these effects pooled.

Table 1 shows the mean ear emergence times of each genotype, measured from the sowing date. Clearly there are large differences between the $5 \mathrm{~A}$ chromosomes with respect to their effect on ear emergence on a Chinese Spring background. The analysis of variance of these data (table 2) confirms this and further shows that significant differences are exhibited both between the substitution lines and the hybrids, and between the means of the two groups.

\section{TABLE 1}

Mean ear emergence times (days from sowing)

$\begin{array}{lccccc} & \begin{array}{c}\text { Chinese Spring } \\ \text { (CS) }\end{array} & \begin{array}{c}\text { CS (Triticum } \\ \text { spelta 5A) }\end{array} & \text { CS (Hope 5A) } & \text { CS (Timstein } & \text { CS (Cappelle- } \\ & & 52 \cdot 00 & 54 \cdot 56 & 62 \cdot 38 & \text { Desprez 5A) } \\ \text { Chinese Spring } & 64.59 & 45 \cdot 88 & 50 \cdot 00 & 55 \cdot 88 & 67 \cdot 00 \\ \text { CS (Triticum spelta 5A) } & & & 50 \cdot 34 & 53 \cdot 36 & 61 \cdot 31 \\ \text { CS (Hope 5A) } & & & 61 \cdot 92 & 66 \cdot 00 \\ \text { CS (Timstein 5A) } & & & & 72 \cdot 25 \\ \text { CS (Cappelle-Desprez 5A) } & \text { Parental mean }=60 \cdot 63 . & \text { Hybrid mean }=58 \cdot 80 .\end{array}$

TABLE 2

Analysis of variance

$\begin{array}{crcc}\text { Item } & \text { d.f. } & \text { M.S. } & \text { P } \\ \text { Between genotypes } & 14 & 703.39 & <0.001 \\ \text { Between parental lines } & 4 & 1305.53 & <0.001 \\ \text { Between hybrids } & 9 & 498.53 & <0.001 \\ \text { Parental lines } v \text {. Hybrids } & 1 & 138.59 & <0.001 \\ \text { Pooled Error } & 181 & 4.70 & \\ \text { Total } & 195 & & \end{array}$


These results contirm those obtained by Law et al. (loc. cit.) about the differential effects of different $5 \mathrm{~A}$ chromosomes and in the present study the chromosomes may be ranked in order of "earliness" as Triticum spelta 5A, Hope 5A, Timstein 5A, Chinese Spring 5A and Cappelle-Desprez 5A.

The significance of the Parental lines $v$. Hybrids item in the analysis of variance suggests that the hybrids exhibit potence with respect to the " early" chromosomes. The dominance relationships of the different chromosome combinations can be examined in detail by calculating the deviation of each hybrid from its mid-parental value. The values obtained are shown in table 3. Significant potence is exhibited in only three crosses, those of Chinese Spring (CS) $\times$ CS (Triticum spelta $5 \mathrm{~A}$ ), CS $\times$ CS (Hope 5A), and CS (Timstein 5A) $\times$ GS (Hope 5A). In each case potence is in the direction of the early chromosome. This is in agreement with the results of Law et al. (loc. cit.) and Pugsley (1971).

TABLE 3

Potence relationships

Chinese Spring

CS (Tricicum spelta $5 \mathrm{~A}$ )

CS (Hope 5A)

CS (Timstein 5A)

CS (Cappelle-Desprez 5A)

$\begin{array}{cccc}\text { CS (Triticum } & & \text { CS (Timstein } & \text { CS (Cappelle- } \\ \text { spelta 5A) } & \text { CS (Hope 5A) } & 5 \mathrm{~A}) & \text { Desprez 5A) } \\ -3.24 * & -2.90 * & -0.88 & -1.42 \\ & +1.90 & +1.98 & +1.44 \\ & & -2.77 * & +0.02 \\ & & & -1.09\end{array}$

Probability: $*=0 \cdot 05-0 \cdot 01$.

In order to investigate further the relationships between these chromosomes it is possible to set up models including different numbers of factors and to describe each of the parental lines in terms of whether they carry the + (late) or - (early) component of a factor. The validity of such models can be tested by least squares fitting techniques on the generation means.

From the data in table 1 the five $5 \mathrm{~A}$ chromosomes can be roughly classified into three groups, according to their ear emergence times. Triticum spelta and Hope, constitute an "early" group, Timstein and CS an " intermediate", and Cappelle-Desprez a "late" group. Similarly within the first two groups the difference between Triticum spelta and Hope on the one hand, and Timstein and CS on the other are similar in magnitude. Thus it is possible to specify the differences between the five chromosomes in terms of three factors. First a factor whose + and - components specify the difference between the "early" plus "intermediate" genotypes and CappelleDesprez. Secondly a factor whose components specify the difference between the "early" and "intermediate" genotypes; and thirdly a factor whose components specify the differences within the first two groups. If these factors are arbitrarily called $f_{1}, f_{2}$ and $f_{3}$ respectively, then each chromosome can be defined as carrying the + or - components of each factor (table 4). In genetical terms the + and - components can be taken as the two alleles at each of three separate loci, although of course, in the present case they cannot be identified as such. Nevertheless, the above model can be tested by conventional least squares fitting techniques on the observed mean ear emergence times of the five genotypes. Four parameters are necessary to 
parental lines. Equally the small dominance effects would suggest that heterozygous $\times$ heterozygous effects would not be large. However homozygous $\times$ heterozygous $(j)$ interactions may be the cause of failure of the model.

Models of different " $j$ " type interactions were fitted to the data and a minimum of three extra parameters was needed to sufficiently explain the observed variation (table 6). This ten-parameter model gives a satisfactory fit to the observed data.

TABLE 6

Model fiting on parental lines and hybrids

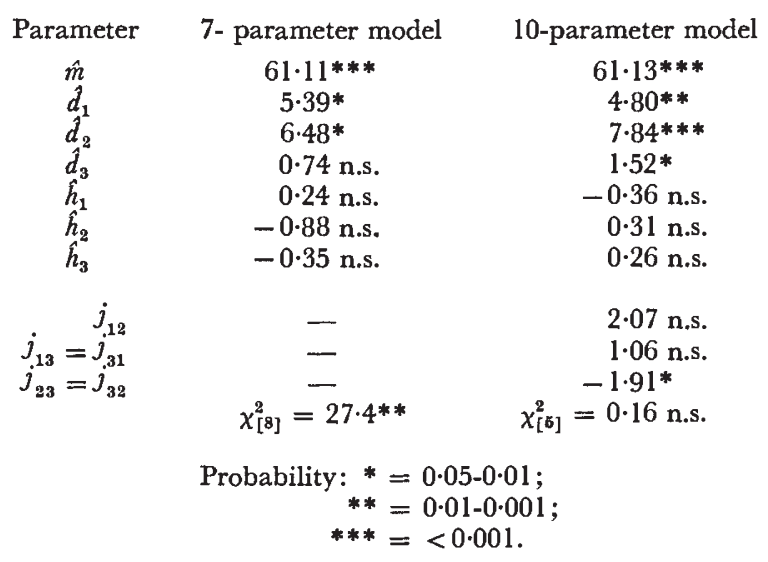

\section{Discussion}

The three factor model proposed provides a satisfactory explanation of the "multiple allelism" of these 5A chromosomes from different varieties. Interestingly, on this model, two crosses, namely $\mathrm{CS} \times \mathrm{CS}$ (Triticum spelta $5 \mathrm{~A}$ ) and CS (Hope 5A) $\times$ CS (Timstein 5A) have the same genetical expectations. In fact, the analysis of variance of the raw data for these two crosses fails to detect any difference between them.

However it is not possible from these data to discover whether the three factors are three separate loci or three components of the same locus, for example different mutational sites. To do this it is necessary to examine segregants from these hybrids. A particularly sensitive method of detecting recombination was used by Law et al. (loc. cit.) where the recipient monosomic for the chromosome in question was used to derive homozygous products from hybrids between CS and CS (Hope 5A). They detected no recombinants but as can be seen from table 4 , none are expected on the present model since these lines only differ by the factor $f_{2}$. To detect recombination requires, of course, that the parental lines differ by at least two factors and the cross of CS (Cappelle-Desprez 5A) $\times$ CS (Hope 5A) should be particularly efficient in this respect since recombinant lines containing all three + components are expected. Such lines would be later than either the parental lines or the hybrid, and chromosomes of this type may already exist in extreme winter wheats.

On the model proposed here the Triticum spelta $5 \mathrm{~A}$ is the "earliest" possible chromosome. This chromosome would be particularly potent if transferred into winter wheats, transforming them into spring-type wheats 
because of their much reduced vernalisation requirement. This can easily be done using the chromosome substitution techniques described by Law and Worland (1973) and may have important consequences in plant breeding and also in providing valuable stocks for looking at the underlying physiological and developmental processes associated with ear emergence and vernalisation requirement.

The variation between the different $5 \mathrm{~A}$ chromosomes contrasts with the variation found for chromosome $5 \mathrm{D}$ by Law et al. (loc. cit.), where they detected fewer variants. However their evidence of the homology of the genes $V r n 1$ and $V r n 3$ on 5A and 5D through their use of alien substitution lines would suggest that similar variation to the $5 \mathrm{~A}$ variation may be found for 5D. They also suggested that CS (Hope 5D) was equivalent to CS (Cappelle-Desprez 5A), and CS5D was an " early" chromosome equivalent to either Hope 5A or T. spelta 5A. If this is so, then recombinants should have been observed from the cross of CS with CS (Hope 5D) if the three factors hypothesised in this paper are distinct and easily separable loci. No recombinants were found from this cross (Law et al., loc. cit), suggesting that the genetic description of the variation on chromosome $5 \mathrm{~A}$ relates to either a tightly linked group of genes or different components of the $V r n 1$ locus. The genetic validity of the three factor model may thus be difficult to establish except that it may be possible to identify the different variants predicted by the model among other substitution lines of chromosome 5A in CS.

Using the wide variation evidently available on chromosome $5 \mathrm{~A}$ and $5 \mathrm{D}$ it should be possible to finely adjust the ear emergence times of a spring sown variety to achieve close adaptation to different environments. Similar adaptation of winter sown varieties to different vernalisation treatments should also be possible so that a wide adaptation to different environmental conditions could be achieved using one basic variety if desired.

\section{REFERENCES}

AKeRMAN, A., AND MACKey, J. 1949. Efforts to increase yield of spring wheat. II. Crosses between spring and winter wheats: description of Svälot Ello spring wheat. Sveriges Utsädesfar. tidskr., 59, 105-117.

HALLORAN, G. M., AND BOYDELL, C. W. 1967. Wheat chromosomes with genes for vernalisation response. Can. 7. Genet. Cytol., 9, 632-639.

KUSPIRA, J., AND UNRAU, J. 1957. Genetic analysis of certain characters in common wheat using whole chromosome substitution lines. Can. F. Plant. Sci., 37, 300-326.

LAW, c. N. 1968. Genetic analysis using inter-varietal chromosome substitutions. Proc. 3rd Int. Wheat Genet. Symp., Canberra, 331-342.

LAW, C. N. 1972. The analysis of inter-varietal chromosome substitutions in wheat and their first generation hybrids. Heredity, 28, 169-179.

LAW, C. N., AND WORLAND, A. J. 1973. Aneuploidy in wheat and its uses in genetic analysis. Annual Rep. Plant Breeding Institute, 1972, 25-65.

LAW, C. N., WORLAND, A. J., AND GIORGI, B. 1976. The genetic control of ear emergence by chromosomes 5A and 5D of wheat. Heredity, 36, 49-58.

MATHer, K., AND JINKs, J. L. 1971. Biometrical Genetics. Chapman and Hall Ltd., London.

MAYSTRENKO, O. I. 1974. Identification of chromosomes carrying genes $V r n 1$ and $\operatorname{Vrn} 3$ inhibiting winter habit in wheat. European Wheat Aneuploid Neresletter, 4, 49-52.

PUGSLEY, A. T. 1971. A genetic analysis of the spring-winter habit of growth in wheat. Aust. F. Agric. Res., 22, 21-31.

PUGSLEY, A. T. 1972. Additional genes inhibiting winter habit in wheat. Euphytica, 21, $547-552$.

UNRAU, J. 1950. The use of monosomics and nullisomics in cytogenetical studies of common wheat. Sci. Agric., 30, 66-89. 